\title{
Evaluation Standard for Safety Coefficient of Roller Compacted Concrete Dam Based on Finite Element Method
}

\author{
Bo Li, ${ }^{1}$ Zhijun Zhang, ${ }^{2}$ Yan Liu, ${ }^{3}$ and Shengmei Yang ${ }^{1}$ \\ ${ }^{1}$ Engineering Safety and Disaster Prevention Department, Changjiang River Scientific Research Institute, Wuhan 430010, China \\ ${ }^{2}$ Construction Design Department, Changjiang Institute of Survey, Planning, Design and Research, Wuhan 430010, China \\ ${ }^{3}$ Center of Construction Management \& Quality \& Safety Supervision, MWR, Beijing 100038, China \\ Correspondence should be addressed to Bo Li; 1b007403@163.com
}

Received 16 March 2014; Revised 27 May 2014; Accepted 10 June 2014; Published 6 July 2014

Academic Editor: Yan-Jun Liu

Copyright (c) $2014 \mathrm{Bo} \mathrm{Li}$ et al. This is an open access article distributed under the Creative Commons Attribution License, which permits unrestricted use, distribution, and reproduction in any medium, provided the original work is properly cited.

\begin{abstract}
The lack of evaluation standard for safety coefficient based on finite element method (FEM) limits the wide application of FEM in roller compacted concrete dam (RCCD). In this paper, the strength reserve factor (SRF) method is adopted to simulate gradual failure and possible unstable modes of RCCD system. The entropy theory and catastrophe theory are used to obtain the ultimate bearing resistance and failure criterion of the RCCD. The most dangerous sliding plane for RCCD failure is found using the Latin hypercube sampling (LHS) and auxiliary analysis of partial least squares regression (PLSR). Finally a method for determining the evaluation standard of RCCD safety coefficient based on FEM is put forward using least squares support vector machines (LSSVM) and particle swarm optimization (PSO). The proposed method is applied to safety coefficient analysis of the Longtan RCCD in China. The calculation shows that RCCD failure is closely related to RCCD interface strength, and the Longtan RCCD is safe in the design condition. Considering RCCD failure characteristic and combining the advantages of several excellent algorithms, the proposed method determines the evaluation standard for safety coefficient of RCCD based on FEM for the first time and can be popularized to any RCCD.
\end{abstract}

\section{Introduction}

Roller compacted concrete dams (RCCDs) are now constructed with great frequency in the world. Over the past two decades, many high RCCDs have been built, such as the $130 \mathrm{~m}$ high Baise dam and the $200.5 \mathrm{~m}$ high Guangzhao dam in China. Currently the highest dam of this type is Longtan Dam at $216 \mathrm{~m}$ in China, with Diamer-Bhasha Dam planned at $272 \mathrm{~m}$ in Pakistan [1].

The RCCD is mainly composed of the body and interface. The interface plays an important role as the weak point of the stability of the RCCD. The stability and safety of a RCCD to guard against sliding along the dam interface are key factors that must be addressed in the design stage of the RCCD. Generally speaking, analysis methods for the RCCD stability should include the rigid-body limiting equilibrium method (LEM) and finite element method (FEM). LEM is normally adopted before considering FEM. Ministry of Water Resources [2] presents evaluation standards for the LEM.
Compared with the LEM, the FEM used in the calculation of antisliding analysis does not require any assumption of the sliding plane, but safety coefficient based on FEM does not have corresponding evaluation standard, which limits the usage of FEM in RCCD. In order to popularize FEM to RCCD more widely than ever, it is necessary to study the evaluation standard for RCCD safety coefficient based on FEM.

When the dam is in critical state, small perturbation will lead the dam away from the equilibrium state into the instability quickly. According to the dissipative structure theory, in the course of dam failure, some abnormal deformations appear certainly with the reduction of system entropy [3]. Thereby, in this paper, RCCD failure criterion is put forward combining the entropy theory and catastrophe theory.

RCCD failure is formed under a variety of combined factors. The sliding mechanism and failure mode are determined by the mechanical parameters (deformation and strength parameters) of RCCD interface. The sensitivity analysis of effect factors for the stability of RCCD interface can 
determine the major control plane of the RCCD, namely, the most dangerous sliding plane which has great significance for RCCD antisliding stability analysis. In this paper, sensitivity analysis for RCCD interface stability based on the RCCD failure criterion with a hybrid algorithm is proposed. Latin hypercube sampling (LHS) was introduced by McKay et al. [4] as a means of generating a distribution of plausible collections of parameter values from a multidimensional distribution and has been further developed for different purposes by several researchers $[5,6]$. Partial least squares regression (PLSR) is a multivariate statistical data analysis method, which can establish effectively the model for the data involving multicollinearity or small sample size $[7,8]$. In order to combine the advantages of LHS and PLSR, a hybrid algorithm which combines LHS with auxiliary analysis of PLSR is proposed for the sensitivity analysis. Through the sensitivity analysis of effect factors, the most dangerous sliding plane of RCCD failure is determined. On this basis, the evaluation standard for RCCD safety coefficient based on FEM is proposed with a hybrid optimization algorithm. Least squares support vector machines (LSSVM) are least squares versions of support vector machines (SVM), which are a set of related supervised learning methods that analyze data and recognize patterns and are used for classification and regression analysis [9]. Particle swarm optimization (PSO) is originally attributed to Kennedy and Eberhart who were illumed by the behavior of bird swarms in 1995 [10]. It has been demonstrated that PSO outperforms genetic algorithm in multivariable function optimization [11]. A hybrid optimization algorithm which combines LHS, LSSVM, and PSO is proposed for evaluation standard of RCCD safety coefficient based on FEM.

This paper reports the analyses of evaluation standard for safety coefficient of the Longtan RCCD based on FEM. The result shows that the Longtan RCCD is safe in the design condition.

\section{RCCD Failure Criterion}

2.1. RCC Yield Criterion. The transversely isotropic and elastoplastic model is used for the constitutive relationship of RCCD material. The isotropic and elastoplastic model is used for the constitutive relationship of the rock material of the dam foundation. The most typical Drucker-Prager (D-P) criterion $[12,13]$ in geotechnical engineering is used for the yield criterion of the RCCD and rock of the dam foundation:

$$
F=\alpha I_{1}+\sqrt{J_{2}}-K=0,
$$

where $I_{1}$ and $J_{2}$ are the first invariant of stress tensor and the second invariant of deviatoric stress tensor, respectively. Both $\alpha$ and $K$ are strength constants, which are related to cohesion $c$ and internal friction angle $\varphi$. Different yield criterions can be realized in finite element calculations through changing the expressions of $\alpha$ and $K$. In this paper, the D-P criterion of equivalent-area cones is used.

2.2. Strength Reserve Factor Method. Zienkiewicz and Pande [14] firstly introduced strength reserve factor (SRF) method to elastoplastic FEM analysis. The dam and dam foundation have strength reserve ability. When shear strength parameters (cohesion $c$ and internal friction coefficient $f$ ) of the dam decrease, the dam will lose strength and become instable. Basic principle of SRF method is that the cohesion $c$ and internal friction coefficient $f$ are divided by the reduction coefficient $K_{R}$ :

$$
c_{R}=\frac{c}{K_{R}}, \quad f_{R}=\frac{f}{K_{R}} .
$$

With the gradual increasing of the reduction coefficient $K_{R}$, the process of RCCD failure can be obtained, and the dam will reach a limit state.

2.3. Entropy Catastrophe Criterion of RCCD Failure. The process of RCCD failure is the evolution process from the "disorder" to the "order." Because of the correspondence between the system entropy and the system disorder degree, the safety state of RCCD can be easily judged by calculating the entropy value of RCCD in this process [3].

The information entropy is a measure of the disorder associated with a random variable. The concept of information entropy was introduced by Shannon [15], which is computed with the algorithm

$$
S=-\phi \sum_{i}^{n} p_{i} \log _{2} p_{i}
$$

where $S$ is the information entropy and $p_{i}$ is the probability of a discrete random variable.

In the process of RCCD failure, the dam interface is main control plane. Therefore, the interface entropy is mainly researched here. The total displacement $U$ of the interfaces can be expressed as follows:

$$
\begin{aligned}
& U=\sum_{i}^{n} u_{i}, \quad(i=1,2, \ldots, n), \\
& \lambda_{i}=\frac{u_{i}}{U}, \quad(i=1,2, \ldots, n),
\end{aligned}
$$

where $u_{i}$ is the displacement of the element (FEM element) $i$ and $\lambda_{i}$ expresses the distribution of the interface displacement. Obviously, the physical variable $\lambda_{i}$ has nonnegative characteristic, which is the same as the probability mass function. According to (3), the information entropy $S$ of the interface displacements is defined as follows:

$$
S=-\phi \sum_{i}^{n} \lambda_{i} \ln \lambda_{i}
$$

With the reduction of the strength parameters by SRF method, the degree of order increases, and the entropy decreases. When the entropy decreases to certain value, the instable interface will be completely damaged.

Because the entropy $S$ changes with the reduction coefficient $K_{R}$, it can be shown as follows:

$$
S^{(1)}(x)=a_{0}+a_{1} x+a_{2} x^{2}+a_{3} x^{3}+\cdots+a_{n} x^{n},
$$


where $a_{0}, a_{1}, \ldots, a_{n}$ are undetermined coefficients and $x$ is the reduction coefficient $K_{R}$. Using multiple regression analysis, the coefficients $a_{0}, a_{1}, \ldots, a_{5}$ can be obtained.

The derivation of (6) gets the following equation:

$$
V=a_{1}+2 a_{2} x+3 a_{3} x^{2}+4 a_{4} x^{3}+5 a_{5} x^{4},
$$

where $V$ is the potential function; when $b_{0}=a_{1}, b_{1}=2 a_{2}$, $b_{2}=3 a_{3}, b_{3}=4 a_{4}$, and $b_{4}=5 a_{5}, V$ can be expressed as

$$
V=b_{0}+b_{1} x+b_{2} x^{2}+b_{3} x^{3}+b_{4} x^{4}
$$

When $p=b_{3} / 4 b_{4}, x=y-p$, the cube is eliminated:

$$
V=c_{4} y^{4}+c_{2} y^{2}+c_{1} y+c_{0}
$$

where $c_{4}=b_{4}, c_{2}=6 p^{2} b_{4}-3 p b_{3}+b_{2}, c_{1}=-4 p^{3} b_{4}+3 p^{2} b_{3}-$ $2 p b_{2}+b_{1}$, and $c_{0}=p^{4} b_{4}-p^{3} b_{3}+p^{2} b_{2}-p b_{1}+b_{0}$. When $y=\left(Z / \sqrt[4]{4 c_{4}}\right)\left(c_{4}>0\right)$ or $y=\left(Z / \sqrt[4]{-4 c_{4}}\right)\left(c_{4}<0\right)$, (9) can be written as follows:

$$
V=\frac{1}{4} Z^{4}+\frac{1}{2} u Z^{2}+v Z
$$

or

$$
V=-\frac{1}{4} Z^{4}+\frac{1}{2} u Z^{2}+v Z
$$

where $u=c_{2} / \sqrt{\left|c_{4}\right|}, v=c_{1} / \sqrt[4]{4\left|c_{4}\right|}$.

Equation (10) is the standard potential function of regular cusp catastrophe. Equation (11) is the standard potential function of dual cusp catastrophe $[16,17]$.

When $\partial V / \partial Z=0,(10)$ and (11) can be expressed, respectively, as follows:

$$
\begin{aligned}
& Z^{3}+u Z+v=0, \quad \text { (Regular cusp catastrophe) } \\
& -Z^{3}+u Z+v=0, \quad \text { (Dual cusp catastrophe). }
\end{aligned}
$$

When $\partial^{2} V / \partial Z^{2}=0,(10)$ and (11) can be expressed, respectively, as follows:

$$
\begin{aligned}
& 3 Z^{2}+u=0, \quad \text { (Regular cusp catastrophe) } \\
& -3 Z^{2}+u=0, \quad \text { (Dual cusp catastrophe). }
\end{aligned}
$$

Through (12) and (14) or (13) and (15), bifurcation set equation is shown as

$$
\Delta=4 u^{3}+27 v^{2}=0 .
$$

The RCCD failure criterion is defined as follows.

If $\Delta=4 u^{3}+27 v^{2}>0$, the RCCD is in the stable state.

If $\Delta=4 u^{3}+27 v^{2}=0$, the RCCD is in the critical state between stability and failure.

If $\Delta=4 u^{3}+27 v^{2}<0$, the RCCD is in the failure state.

\section{Determination of the Most Dangerous Sliding Plane of RCCD Failure}

In this paper, the LHS method is adopted to generate the combinations of various mechanical parameters, and the elastoplastic finite element analysis is carried out based on the entropy catastrophe criterion; in the end, the most dangerous sliding plane is obtained by the sensitivity analysis of the mechanical parameters using the auxiliary analysis of PLSR.

3.1. Latin Hypercube Sampling. The LHS technique is a constrained sampling technique whereby the input parameter range is divided into equiprobable nonoverlapping intervals. Let $N$ denote the required number of realizations and $K$ the number of random variables. An $N \times K$ matrix $P$, in which each of the $K$ columns is a random permutation of $1, \ldots, N$, and an $N \times K$ matrix $R$ of independent random numbers from the uniform $(0,1)$ distribution are established. These matrices form the basic sampling plan represented by the matrix $S$ as

$$
S=\frac{P-R}{N} .
$$

Each element of $S, s_{i j}$, is then mapped according to its target marginal distribution as

$$
\widehat{x}_{i j}=F_{x_{j}}^{-1}\left(s_{i j}\right) \text {, }
$$

where $F_{x_{j}}^{-1}$ represents the inverse of the target cumulative distribution function for variable $j$. A vector $\widehat{X}_{i}=$ $\left[\widehat{x}_{i 1}, \widehat{x}_{i 2}, \ldots, \widehat{x}_{i k}\right]$ now contains input data for one deterministic computation.

\subsection{Partial Least Squares Regression and Auxiliary Analysis}

3.2.1. Partial Least Squares Regression. The basic steps of PLSR are given as follows [18].

Step 1. $E_{0}$ and $F_{0}$ are standardized matrix of independent variable $X$ and dependent variable $y$, respectively.

Step 2. The first principal component $t_{1}$ is extracted from $E_{0}$ : $t_{1}=E_{0} w_{1}, w_{1}=E_{0}^{T} F_{0} /\left\|E_{0}^{T} F_{0}\right\|$, and $\left\|w_{1}\right\|=1$. Two regression equations are solved as follows:

$$
E_{0}=t_{1} p_{1}^{T}+E_{1}, \quad F_{0}=t_{1} r_{1}^{T}+F_{1},
$$

where $E_{1}$ and $F_{1}$ are residual matrix, and regression coefficient can be obtained as follows:

$$
p_{1}=\frac{E_{0}^{T} t_{1}}{\left\|t_{1}\right\|^{2}}, \quad r_{1}=\frac{F_{0}^{T} t_{1}}{\left\|t_{1}\right\|^{2}} .
$$

Step 3. $E_{0}$ and $F_{0}$ are replaced by $E_{1}$ and $F_{1}$, respectively, and the same method is used to calculate $w_{2}$ and $t_{2}$.

It is not necessary to use all principal components to establish PLSR model, but a truncated way is adopted, which uses frontal principal components, $t_{1}, t_{2}, \ldots, t_{m}$, to establish good prediction model. $m$ can usually be obtained by cross validation $(\mathrm{CV})[19]$. 
3.2.2. Auxiliary Analysis. The principal component $t_{h}(h=$ $1,2, \ldots, m)$ is obtained using the PLSR method. On the one hand, the $t_{h}$ as much as possible represents the information of the independent variable $X=\left\{x_{1}, \ldots, x_{p}\right\}$; on the other hand, it explains the dependent variable $y$ to the greatest extent. In fact, the explanation ability of the $t_{h}$ is equivalent to the complex correlation coefficient $R^{2}$ of linear regression equation on $t_{h}$. The explanation abilities of the $t_{h}$ for the $x_{j}(j=1,2, \ldots, p), X$, and $y$ are expressed, respectively, as follows:

$$
\begin{gathered}
R d\left(x_{j} ; t_{h}\right)=R^{2}\left(x_{j}, t_{h}\right), \\
R d\left(X ; t_{h}\right)=\frac{1}{p} \sum_{i=1}^{p} R d\left(x_{j} ; t_{h}\right), \\
R d\left(y ; t_{h}\right)=R^{2}\left(y, t_{h}\right) .
\end{gathered}
$$

The ability that the factor $x_{j}$ explains the variable $y$ is expressed by the variable projection importance index $\operatorname{VIP}_{j}(j=1,2, \ldots, p)$ :

$$
\mathrm{VIP}_{j}=\frac{\sum_{h=1}^{m} R d\left(y ; t_{h}\right) w_{h j}^{2}}{\sum_{h=1}^{m} R d\left(y ; t_{h}\right)},
$$

where $w_{h j}$ is the $j$ th component of the variable $w_{h}$.

\subsection{The Steps of Determining the Most Dangerous Sliding Plane} of the RCCD. The steps of determining the most dangerous sliding plane of the RCCD are shown as follows.

Step 1. The deformation and strength parameters of the RCCD are taken as the effect factors, and the ranges of the factors are determined.

Step 2. The combinations of the effect factors are established by the LHS.

Step 3. The elastoplastic finite element model is established, and the safety coefficients of the dam failure corresponding to the combinations in Step 2 are calculated using RCCD failure criterion.

Step 4. The sensitivities of mechanical parameters to the whole stability of the dam are determined using the auxiliary analysis of PLSR to analyze the combinations of the effect factors and corresponding safety coefficients of the dam failure. The interface of the most sensitive parameter is the most dangerous sliding plane.

\section{Evaluation Standard Analysis of RCCD Safety Coefficient}

4.1. Failure Process Analysis of RCCD Interface. Compared with conventional concrete dam, RCCD has obvious rolled characteristic and relatively weak construction interface. A lot of studies show that at the time of the RCCD failure, an interface is yielded completely, and the sliding channel appears [20]. Figure 1 shows typical failure process of RCCD

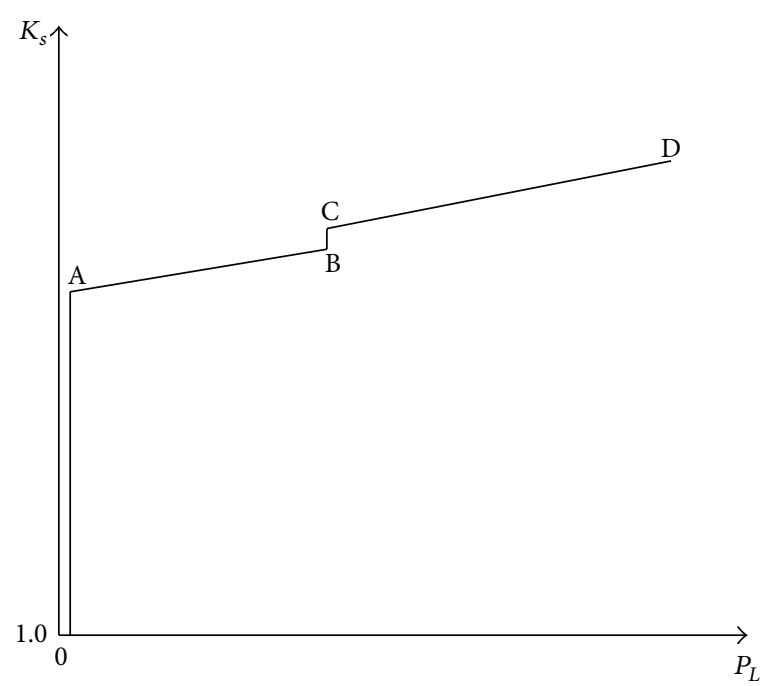

FIGURE 1: Typical failure process of RCCD interface.

interface. $Y$-axis is strength reserve coefficient $K_{s}$ and $X$-axis is the interface yield rate $P_{L}$.

(1) Stability Stage (before Point $A$ ). When $K_{s}=1.0$, in addition to local small yield in the dam, the dam is basically in linear elastic state. With the increase of the $K_{s}$, yield area has a little expansion. Only when the $K_{s}$ increases to certain value (corresponding point A), the yield area begins to expand. The point $\mathrm{A}$ is called quasielastic state.

(2) Yield Deformation Stage (between Point A and Point C). With the increase of the $K_{s}$ from the point A, the yield area increases rapidly to certain scope (about 30\% 50\%). Near the point $\mathrm{B}$, the extension temporarily becomes slow until Point C.

(3) Failure Stage (between Point C and Point D). The yield area expands rapidly from Point $C$. The upstream and downstream yield areas of the interface sharply joint together. Near Point $\mathrm{D}$, sliding channel is formed and whole sliding failure of the dam leads to the loss of the ability of dam operation.

4.2. The Determination of the Evaluation Standard for RCCD Safety Coefficient. In the process of the RCCD failure, the quasielastic state is an important critical point. Before this point, the dam is mainly in elastic state and it is stable as a whole. After this point, the yield area rapidly expands until the dam failure. Therefore, the evaluation standard for RCCD safety coefficient based on the SRF method is given as

$$
K_{e} \geq\left[K_{e}\right]
$$

where $K_{e}$ is the safety coefficient in the quasielastic state and $\left[K_{e}\right]$ is the allowable value of the $K_{e}$.

If the allowable value $\left[K_{e}\right]=1.0$, the dam is very safe in the design condition. But in the process of dam operation, water level overload, work environment worsening, and the variation of mechanical parameters and other adverse factors will appear. Therefore, these factors must be considered into 
the impact of the $\left[K_{e}\right]$. Firstly, according to the results of the test and statistics, the ranges of the factors are obtained. Secondly, when the above factors change in the ranges at the same time, the $\left[K_{e}\right]$ is obtained in the most adverse circumstance where the minimum of the quasielastic strength reserve coefficient appears. Specific expression is given as follows:

$$
\left[K_{e}\right]=1.0 \times\left(1+\frac{\left|K_{d}-K_{m}\right|}{K_{d}}\right),
$$

where $K_{d}$ is the quasielastic strength reserve coefficient in the design condition and $K_{m}$ is the minimum of the quasielastic strength reserve coefficient with the variation of various factors.

Before determining $\left[K_{e}\right]$, the $K_{m}$ must be determined firstly. The stability of RCCD is affected by many factors, which mainly include the strength parameters (internal friction coefficient and cohesion), deformation parameters (elastic modulus), water level, and seepage condition. Among them, the deteriorations of strength parameters and seepage condition and the high water level will make the quasielastic strength reserve coefficient of RCCD become smaller. The effect of the elastic modulus is uncertain to the quasielastic strength reserve coefficient. In this paper, the LHS, LSSVM, and PSO are introduced to solve jointly the $\left[K_{e}\right]$.

\subsubsection{Regression Algorithm of Least Squares Support Vector} Machine. Least squares support vector machine (LSSVM) is a modified version of standard SVM, in which analytical solutions can be obtained solving linear equations instead of a quadratic programming $(\mathrm{QP})$ problem. Given a training data set $\left\{x_{i}, y_{i}\right\}_{i=1}^{N}$, the objective function of optimization problem is defined as $[19,21]$

$$
\min _{w, b, e} \frac{1}{2}\|w\|^{2}+\gamma \frac{1}{2} \sum_{i=1}^{N} e_{i}^{2}
$$

where $w$ is the vector of weights, $\gamma$ is a regularization parameter, and $e_{i}$ is the random error.

The SVM inequality constraint is translated into equality constraint as follows:

$$
y_{i}=w \varphi\left(x_{i}\right)+b+e_{i}, \quad i=1,2, \ldots, N,
$$

where $w$ is a weight vector, $b$ is a bias term, and $\varphi$ is a nonlinear function that map the input pattern $x_{i}$ into a higher-dimensional feature space.

The solution of LSSVM regression will be obtained by constructing Lagrangian function as follows:

$$
\begin{aligned}
L(w, b, e, a)= & \frac{1}{2}\|w\|^{2}+\gamma \frac{1}{2} \sum_{i=1}^{N} e_{i}^{2} \\
& -\sum_{i=1}^{N} a_{i}\left\{w \varphi\left(x_{i}\right)+b+e_{i}-y_{i}\right\},
\end{aligned}
$$

where $a_{i}$ is Lagrangian multiplier. The conditions for optimality are given by

$$
\frac{\partial L}{\partial w}=0, \quad \frac{\partial L}{\partial b}=0, \quad \frac{\partial L}{\partial e_{i}}=0, \quad \frac{\partial L}{\partial a_{i}}=0 .
$$

The solution of (28) is obtained as follows:

$$
\begin{gathered}
w=\sum_{i=1}^{N} a_{i} \varphi\left(x_{i}\right), \quad \sum_{i=1}^{N} a_{i}=0, \quad a_{i}=\gamma e_{i}, \\
w \cdot \varphi\left(x_{i}\right)+b+e_{i}-y_{i}=0 .
\end{gathered}
$$

The Elimination of $w$ and $e$ will yield a linear system instead of a quadratic programming problem:

$$
\left[\begin{array}{cc}
0 & 1_{N}^{T} \\
1_{N}^{T} & Z Z^{T}+\gamma^{-1} I_{N}
\end{array}\right]\left[\begin{array}{l}
b \\
a
\end{array}\right]=\left[\begin{array}{l}
0 \\
y
\end{array}\right]
$$

where $y=\left[y_{1}, y_{2}, \ldots, y_{N}\right]^{T}, 1_{N}=[1, \ldots, 1]^{T}, a=$ $\left[a_{1}, a_{2}, \ldots, a_{N}\right]^{T}, Z=\left[\varphi\left(x_{1}\right), \varphi\left(x_{2}\right), \ldots, \varphi\left(x_{N}\right)\right]^{T}$, and $I_{N}$ is $N \times N$ identity matrix.

LSSVM function can be expressed as follows:

$$
f(x)=\sum_{i=1}^{N} a_{i}\left(\varphi(x) \varphi\left(x_{i}\right)\right)+b .
$$

In order to avoid "dimension disaster" in highdimensional feature space, kernel $k\left(x, x_{i}\right)$ of input space is applied to the inner product of high-dimensional space, which solves cleverly the problem of high-dimensional calculation. Nonlinear function can be finally obtained as follows:

$$
f(x)=\sum_{i=1}^{l} a_{i} k\left(x, x_{i}\right)+b
$$

The kernel function has the following choices: polynomial function, radial basis function (RBF), and sigmoid function. In this paper, $\mathrm{RBF}$ is selected as follows:

$$
k\left(x, x_{j}\right)=\exp \left\{\frac{-\left\|x-x_{j}\right\|^{2}}{2 \sigma^{2}}\right\}
$$

where $\sigma$ is a constant, which determines the scaling of the inputs in the RBF.

Main parameters of LSSVM using RBF are regularization parameter $\gamma$ and kernel function width $\sigma$. To a great extent, the two parameters determine learning and generalization ability of LSSVM. Grid search algorithm is usually applied to determine these two parameters. In order to overcome the computational complexity of this method, improved grid search algorithm is applied to determine the parameters in this paper. That is, firstly rough search is applied to determine the best combination using a big step size, and then more precise search is used around this combination by small step size. For each grid point, the mean square error (MSE) from $K$-fold cross validation is determined, and minimum MSE is detected. In $K$-fold cross validation, a single subsample is used as the testing data for the LSSVM model, and the remaining subsamples are used as training data. The cross validation process is run repeatedly, with each subsample used exactly once as the testing data. 
Specific steps are shown as follows.

Step 1. Determine the ranges of the regularization parameter $\gamma$ and kernel function width $\sigma: \gamma \in\left[2^{-10}, 2^{-18}, \ldots, 2^{10}\right], \sigma \in$ $\left[2^{-10}, 2^{-18}, \ldots, 2^{8}\right]$.

Step 2. Normalize training set and use the cross validation of 1-fold to obtain the the best combination of the parameters without affecting model accuracy.

Step 3. Enlarge the ranges of the parameters obtained in Step 2 and use finer grid search to obtain the highest precision combination of parameters by step size $2^{0.5}$.

Compared to general grid search method, improved grid search method not only can reduce training volume, but also can improve forecast accuracy of the model.

4.2.2. Particle Swarm Optimization. PSO is a heuristic global optimization algorithm and has been broadly applied in optimization problems. PSO is developed on a very simple theoretical framework and can be implemented easily with only primitive mathematical operators [22]. In PSO, a group of particles are composed by $m$ particles in $D$ dimension space, where the position of the particle $i$ is $X_{i}=$ $\left(x_{i 1}, x_{i 2}, \ldots, x_{i D}\right)$ and the speed is $V_{i}=\left(v_{i 1}, v_{i 2}, \ldots, v_{i D}\right)$. The speed and position of each particle are changed in accordance with the following equation:

$$
\begin{gathered}
v_{i d}^{j+1}=w_{i} v_{i d}^{j}+c_{1} r_{1}\left(p_{i d}^{j}-x_{i d}^{j}\right)+c_{2} r_{2}\left(p_{g d}^{j}-x_{i d}^{j}\right), \\
x_{i d}^{j+1}=x_{i d}^{j}+v_{i d}^{j+1},
\end{gathered}
$$

where $i=1,2, \ldots, m, d=1,2, \ldots, D$, and $m$ is particle size. $p_{i d}^{j}$ is the $d$ dimension component of the pbest that is individual optimal location of the particle $i$ in the $j$ th iteration. $p_{g d}^{j}$ is the $d$ dimension component of the gbest that is optimal position of all particles in the jth iteration. $w_{i}$ is the inertia weight coefficient. $c_{1}$ and $c_{2}$ are learning factors, which are commonly chosen to be 2 and will also be used in this study. $r_{1}$ and $r_{2}$ are random numbers in the range $[0,1]$.

The inertia weight $w$ is critical for the performance of PSO, which balances global and local exploitation abilities of the swarm. The most well-known algorithm for controlling inertia weight is linearly decreasing inertia weight method [23]. The strategy of linearly decreasing weight is most commonly used, and it can improve the performance of PSO to some extent, but there are still some problems. Therefore, some adaptive algorithms for tuning inertia weight were presented [24, 25]. In this work, we propose nonlinearly decreasing inertia weight method of tuning the value of $w$ for further performance improvement as follows:

$$
w=w_{\max }-\frac{\left(w_{\max }-w_{\min }\right) \times(t-1)^{2}}{\left(t_{\max }-1\right)^{2}}
$$

where $w_{\max }$ and $w_{\min }$ are the maximum and minimum of $w$, respectively, $t$ is the current iteration number, and $t_{\max }$ is the maximum iteration number.
4.2.3. Basic Steps. The basic steps of determining the $\left[K_{e}\right]$ are shown as follows.

Step 1. The most dangerous sliding plane of the RCCD is determined.

Step 2. The elastoplastic model is established, and the quasielastic strength reserve coefficient $K_{d}$ in the design condition is obtained using the SRF method.

Step 3. According to the test result, the ranges of all factors are determined. On this basis, as for the factors which have an unambiguous effect on the dam stability, their values are the maximums or minimums. As for ambiguous factors, LHS is used to construct the combinations of various factors.

Step 4. The quasielastic strength reserve coefficients corresponding to the combinations constructed in Step 3 are calculated using the SRF method.

Step 5. The combinations of various factors and the corresponding quasielastic strength reserve coefficients in Step 4 are trained using LSSVM.

Step 6. The LSSVM model established in Step 5 is optimized using PSO, and the corresponding minimum of quasielastic strength reserve coefficient $K_{m}$ is obtained.

Step 7. According to (24), the $\left[K_{e}\right]$ is obtained.

\section{Example Analysis}

5.1. Establishment of Finite Element Model. The Longtan dam, the highest RCCD in the world currently, was built on the Hongshui River in China. Longtan RCCD was constructed in two phases. In the first phase, normal water level is $375 \mathrm{~m}$, and the elevation of dam top is $382 \mathrm{~m}$. In the second phase, normal water level is $400 \mathrm{~m}$, and the elevation of dam top is $406.5 \mathrm{~m}$. In this paper, $11^{\#}$ dam section of right bank is selected in the first phase. The material partitions of Longtan $11^{\#}$ dam section are shown in Figure 2. According to the material partitions, RCC1, RCC2, RCC3, and foundation interfaces are looked at as main interfaces. The FEM grid of Longtan $11^{\#}$ dam section is shown in Figure 3.

5.2. Determination of the Most Dangerous Sliding Plane of the Dam Failure. In this paper, RCC is regarded as transversely isotropic material. According to the material partitions shown in Figure 2, there are eight groups of parameters that need to be analyzed. The horizontal and vertical elastic modulus of RCC1, RCC2, and RCC3 interfaces are $E_{l i}$ and $E_{v i}(i=1,2,3)$, respectively, the internal friction coefficient and cohesion of RCC1, RCC2, and RCC3 interfaces are $f_{i}$ and $c_{i}(i=1,2,3)$, respectively, the horizontal and vertical elastic modulus of foundation interface are $E_{l j}$ and $E_{v j}(j=4)$, respectively, and the internal friction coefficient and cohesion of foundation interface are $f_{j}$ and $c_{j}(j=4)$, respectively. According to the test result, the ranges of the mechanical parameters of the interfaces are shown in Tables 1 and 2 . 


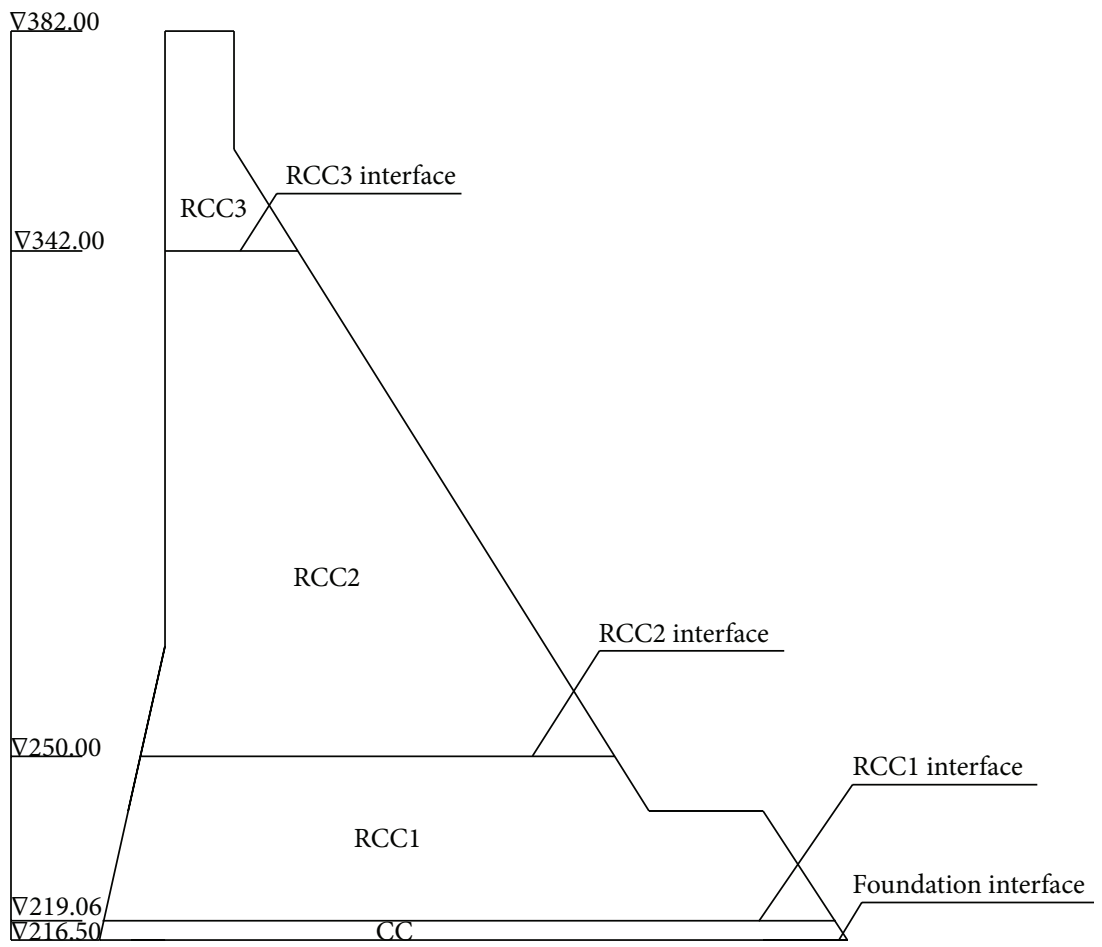

FIgURE 2: Material partitions of Longtan $11^{\#}$ dam section.

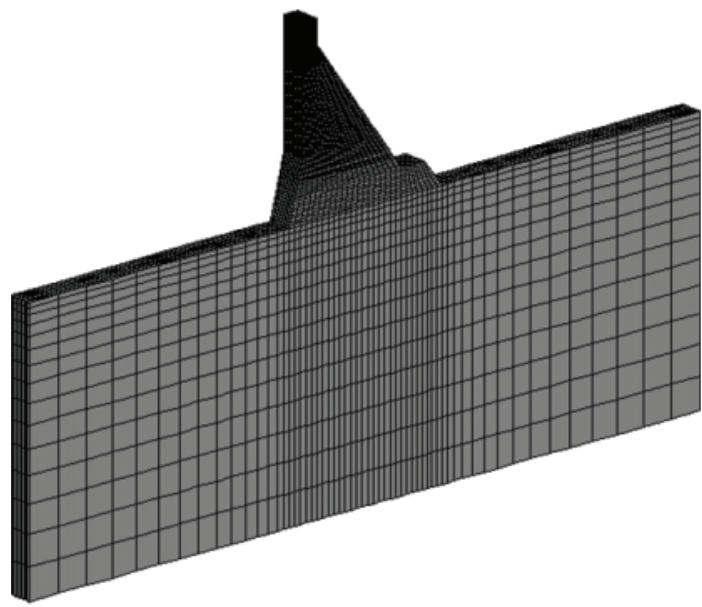

Figure 3: The FEM grid of Longtan $11^{\#}$ dam section.

The design values of main mechanical parameters are shown in Tables 3 and 4 , where $E_{l k}$ and $E_{v k}(k=5,6,7)$ are the horizontal and vertical elastic modulus of RCC1, RCC2, and RCC3 bodies, respectively, $f_{k}$ and $c_{k}(k=5,6,7)$ are the internal friction coefficient and cohesion of RCC1, RCC2, and RCC3 bodies, respectively, $E_{8}$ and $E_{9}$ are the elastic modulus of the conventional concrete and dam foundation rock, respectively, and $f_{8}$ and $c_{8}$ are the internal friction coefficient and cohesion of the conventional concrete and dam foundation rock, respectively.

Using LHS to generate the combinations of the interface mechanical parameters and considering the horizontal elastic

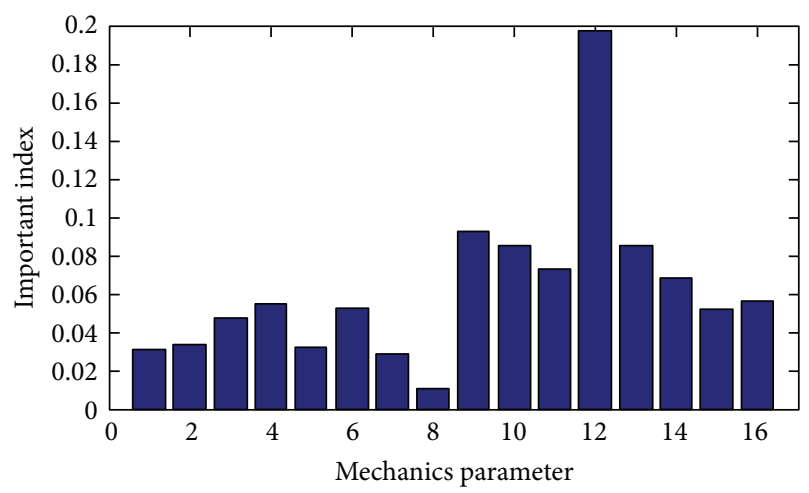

FIGURE 4: The sensibilities of the mechanical parameters to the strength reserve coefficient of the dam failure.

modulus bigger than the vertical, the sample results of the mechanical parameters are obtained in Table 3. Other mechanical parameters adopt design values.

Outer loads are mainly the weight load, water load, and uplift pressure. In this example, the upstream water level is the normal water level $375.00 \mathrm{~m}$, and the downstream water level is $225.00 \mathrm{~m}$. The strength reserve coefficients $K_{u}$ of the dam failure corresponding to the combinations of the mechanical parameters are obtained using the entropy catastrophe criterion. The final results are shown in Table 3.

On this basis, using the auxiliary analysis of PLSR, the important indexes of the mechanical parameters are obtained, and the sensibilities of the mechanical parameters to the $K_{u}$ are shown in Figure 4 . In Figure 4, the $X$-axis is 
TABLE 1: Ranges of interface elastic modulus.

\begin{tabular}{lcccccccc}
\hline Elastic modulus $(\mathrm{GPa})$ & $E_{l 1}$ & $E_{v 1}$ & $E_{l 2}$ & $E_{v 2}$ & $E_{l 3}$ & $E_{v 3}$ & $E_{l 4}$ & $E_{v 4}$ \\
\hline Minimum & 12.19 & 9.75 & 10.56 & 8.45 & 9.75 & 7.80 & 12.20 & 9.75 \\
Maximum & 25.50 & 20.40 & 22.10 & 17.68 & 20.40 & 16.32 & 25.50 & 20.40 \\
\hline
\end{tabular}

TABLE 2: Ranges of interface strength parameters.

\begin{tabular}{lcccccccc}
\hline Strength parameter & $f_{1}$ & $c_{1}(\mathrm{MPa})$ & $f_{2}$ & $c_{2}(\mathrm{MPa})$ & $f_{3}$ & $c_{3}(\mathrm{MPa})$ & $f_{4}$ & $c_{4}(\mathrm{MPa})$ \\
\hline Minimum & 0.82 & 1.17 & 0.73 & 1.04 & 0.70 & 0.66 & 0.86 & 0.83 \\
Maximum & 1.26 & 2.04 & 1.12 & 1.80 & 1.08 & 1.14 & 1.32 & 1.44 \\
\hline
\end{tabular}

TABLE 3: Sample and calculation results of mechanical parameters.

\begin{tabular}{|c|c|c|c|c|c|c|c|c|c|c|c|c|c|c|c|c|c|}
\hline Number & $\mathrm{r} \begin{array}{c}E_{l 1} \\
(\mathrm{GPa})\end{array}$ & $\begin{array}{l}E_{v 1} \\
(\mathrm{GPa})\end{array}$ & $\begin{array}{c}E_{l 2} \\
(\mathrm{GPa})\end{array}$ & $\begin{array}{c}E_{v 2} \\
(\mathrm{GPa})\end{array}$ & $\begin{array}{c}E_{13} \\
(\mathrm{GPa})\end{array}$ & $\begin{array}{c}E_{v 3} \\
(\mathrm{GPa})\end{array}$ & $\begin{array}{c}E_{14} \\
(\mathrm{GPa})\end{array}$ & $\begin{array}{c}E_{v 4} \\
(\mathrm{GPa})\end{array}$ & $f_{1}$ & $\begin{array}{c}c_{1} \\
(\mathrm{MPa})\end{array}$ & $f_{2}$ & $\begin{array}{c}c_{2} \\
(\mathrm{MPa})\end{array}$ & $f_{3}$ & $\begin{array}{c}c_{3} \\
(\mathrm{MPa})\end{array}$ & $f_{4}$ & $\begin{array}{c}c_{4} \\
(\mathrm{MPa})\end{array}$ & $K_{u}$ \\
\hline & 12.41 & 10.28 & 12.29 & 10.14 & 11.70 & 9.36 & 17.30 & 14.9 & 1.08 & 1.71 & & 1. & & 1. & 1. & 1. & 1.82 \\
\hline & 2.86 & 10.64 & 14.6 & 11.99 & 14.54 & 12.20 & 23.95 & 20.22 & 0.04 & $1 .$. & .84 & I.Jנ & & 0.94 & 1.07 & 1.21 & 1.57 \\
\hline & 3.30 & 11.70 & 16.14 & 13.83 & 17.03 & 14.47 & 15.53 & 14.54 & 1. & 1 & 0.88 & 1. & 0. & 0.96 & 0.94 & 1. & .55 \\
\hline 4 & 13.74 & 12.41 & 18.06 & 15.68 & 19.51 & 7.94 & 21.73 & 19.87 & 0.9 & 1.56 & 0.96 & 1.58 & 1.02 & 1.02 & 0.88 & 1.33 & 1.72 \\
\hline & 14.63 & 14.54 & 11.91 & 11.68 & 15.61 & 14.76 & 25.28 & 13.83 & 1.21 & 1.71 & 0.93 & 1.61 & 0.95 & 110 & 1.14 & 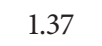 & 1.85 \\
\hline 6 & 19.07 & 9.93 & 7.00 & 9.22 & 1 & 8.2 & 21.73 & & & & & 1. & & & & & 1.89 \\
\hline & 19.95 & 10.28 & 19.22 & 11.07 & 18.8 & 11 & 13.75 & 1 & 0.96 & 3 & 0.81 & 1.4 & 1 & 0.89 & 1.07 & 1.10 & 1.61 \\
\hline o & 21.73 & 13.48 & 14.6 & 8.60 & 17.74 & 11.35 & 23.06 & 11.35 & 1.05 & 1.94 & 0.96 & 1.20 & 1. & 0.92 & 1.14 & 1.12 & 1.72 \\
\hline 9 & 22.62 & 20.22 & 20.75 & 16.30 & 18.45 & 14.19 & 20.85 & 15.25 & 1.02 & 15 & 0.85 & 1.28 & 0.80 & 0.81 & 0.88 & 1.02 & 1.65 \\
\hline 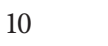 & 23.66 & 17.03 & 12.29 & 8.60 & 19.16 & 13.91 & 24.39 & 15.96 & 0.93 & 2.03 & 1.04 & 1. & 0.87 & 0.96 & 1.05 & 115 & 1.73 \\
\hline 11 & 23.50 & 18.45 & 16.14 & 12.30 & 13.12 & 9.65 & 22.18 & 15.61 & 0.97 & 1.29 & 0.74 & 1.76 & 1.04 & 0.86 & 0.99 & 1.06 & 1.79 \\
\hline 12 & 25.28 & 19.51 & 18.45 & 14.14 & 15.61 & 11.92 & 14.20 & 9.93 & 1.24 & 1.85 & 1.01 & 1.53 & 0.92 & 0.78 & 0.94 & 0.86 & 1.76 \\
\hline
\end{tabular}

TABLE 4: The ranges of the elastic modulus of the RCC bodies, conventional concrete, and dam foundation rock.

\begin{tabular}{lcccccccc}
\hline Elastic modulus $(\mathrm{GPa})$ & $E_{l 5}$ & $E_{v 5}$ & $E_{l 6}$ & $E_{v 6}$ & $E_{l 7}$ & $E_{v 7}$ & $E_{8}$ & $E_{9}$ \\
\hline minimum & 24.38 & 19.50 & 23.44 & 18.75 & 22.50 & 18.00 & 21.38 & 11.20 \\
maximum & 40.95 & 32.76 & 39.38 & 31.50 & 37.80 & 30.24 & 35.91 & 20.80 \\
\hline
\end{tabular}

TABLE 5: Sample and calculation results of mechanical parameters.

\begin{tabular}{lccccccccccccccccc}
\hline \multicolumn{2}{c}{$\begin{array}{c}\text { Number } \\
(\mathrm{GPa})\end{array}$} & $\begin{array}{c}E_{v 1} \\
(\mathrm{GPa})\end{array}$ & $\begin{array}{c}E_{l 2} \\
(\mathrm{GPa})\end{array}$ & $\begin{array}{c}E_{v 2} \\
(\mathrm{GPa})\end{array}$ & $\begin{array}{c}E_{l 3} \\
(\mathrm{GPa})\end{array}$ & $\begin{array}{c}E_{v 3} \\
(\mathrm{GPa})\end{array}$ & $\begin{array}{c}E_{l 4} \\
(\mathrm{GPa})\end{array}$ & $\begin{array}{c}E_{v 4} \\
(\mathrm{GPa})\end{array}$ & $\begin{array}{c}E_{l 5} \\
(\mathrm{GPa})\end{array}$ & $\begin{array}{c}E_{v 5} \\
(\mathrm{GPa})\end{array}$ & $\begin{array}{c}E_{l 6} \\
(\mathrm{GPa})\end{array}$ & $\begin{array}{c}E_{v 6} \\
(\mathrm{GPa})\end{array}$ & $\begin{array}{c}E_{l 7} \\
(\mathrm{GPa})\end{array}$ & $\begin{array}{c}E_{v 7} \\
(\mathrm{GPa})\end{array}$ & $\begin{array}{c}E_{8} \\
(\mathrm{GPa})\end{array}$ & $\begin{array}{c}E_{9} \\
(\mathrm{GPa})\end{array}$ \\
\hline 1 & 23.50 & 10.71 & 14.37 & 9.10 & 19.23 & 10.44 & 23.50 & 16.46 & 31.84 & 22.02 & 25.19 & 23.98 & 30.61 & 18.86 & 30.53 & 14.56 & 1.22 \\
2 & 21.91 & 11.77 & 18.98 & 10.39 & 20.08 & 11.97 & 20.58 & 12.20 & 27.86 & 24.14 & 33.16 & 29.08 & 28.77 & 19.10 & 35.76 & 20.13 & 1.25 \\
3 & 18.98 & 14.12 & 13.91 & 8.91 & 15.18 & 13.34 & 19.51 & 16.03 & 38.80 & 24.41 & 29.66 & 28.31 & 23.27 & 18.61 & 22.11 & 18.78 & 1.24 \\
4 & 17.11 & 11.13 & 21.06 & 11.50 & 19.87 & 10.61 & 24.83 & 17.31 & 35.81 & 31.83 & 37.63 & 28.57 & 35.20 & 24.73 & 23.27 & 12.83 & 1.26 \\
5 & 14.72 & 10.07 & 12.06 & 11.31 & 18.59 & 13.51 & 25.10 & 13.48 & 35.15 & 23.88 & 26.79 & 23.47 & 27.24 & 24.00 & 26.47 & 19.94 & 1.26 \\
6 & 24.30 & 14.97 & 18.75 & 13.53 & 17.95 & 10.78 & 25.37 & 15.39 & 34.82 & 30.24 & 39.22 & 22.19 & 30.30 & 30.12 & 27.34 & 13.98 & 1.27 \\
7 & 20.04 & 17.10 & 11.60 & 10.20 & 11.77 & 8.57 & 17.12 & 16.67 & 39.46 & 30.77 & 36.67 & 27.04 & 30.92 & 28.89 & 28.79 & 19.36 & 1.25 \\
8 & 16.58 & 13.05 & 17.14 & 10.57 & 15.61 & 14.02 & 18.18 & 17.10 & 39.13 & 21.22 & 34.12 & 22.45 & 33.67 & 26.69 & 35.18 & 18.21 & 1.25 \\
9 & 13.65 & 11.35 & 18.52 & 16.66 & 18.38 & 13.85 & 13.93 & 12.63 & 37.80 & 23.35 & 29.34 & 21.17 & 36.12 & 27.18 & 30.82 & 11.30 & 1.28 \\
10 & 16.85 & 12.41 & 20.14 & 14.45 & 10.71 & 7.89 & 22.71 & 14.97 & 25.21 & 29.18 & 28.38 & 21.94 & 34.28 & 21.79 & 29.66 & 14.18 & 1.27 \\
11 & 20.58 & 14.54 & 11.83 & 11.13 & 16.89 & 12.83 & 16.06 & 11.56 & 32.17 & 29.45 & 28.06 & 20.66 & 35.81 & 21.55 & 27.92 & 12.64 & 1.24 \\
12 & 23.77 & 13.90 & 15.52 & 13.71 & 14.54 & 10.10 & 14.99 & 14.54 & 40.45 & 24.94 & 31.57 & 18.88 & 33.98 & 19.59 & 21.53 & 20.70 & 1.28 \\
\hline
\end{tabular}




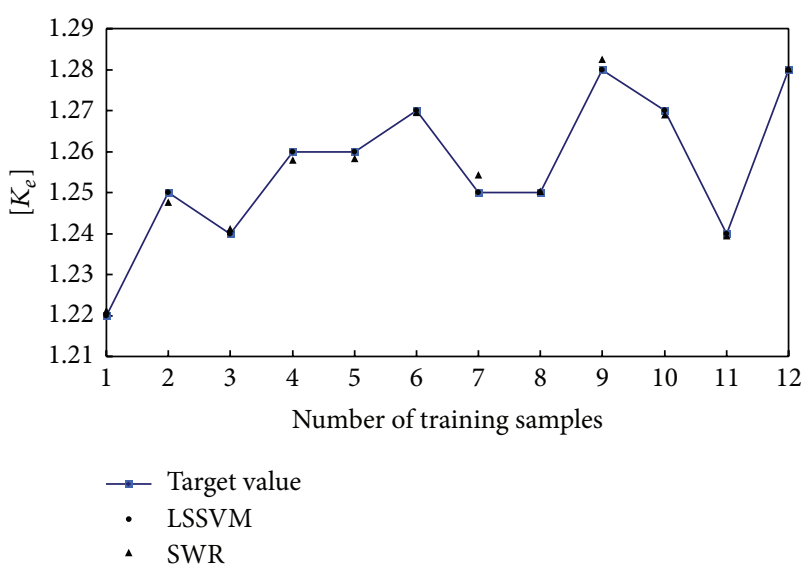

FIGURE 5: Comparison figure of training result of the algorithms.

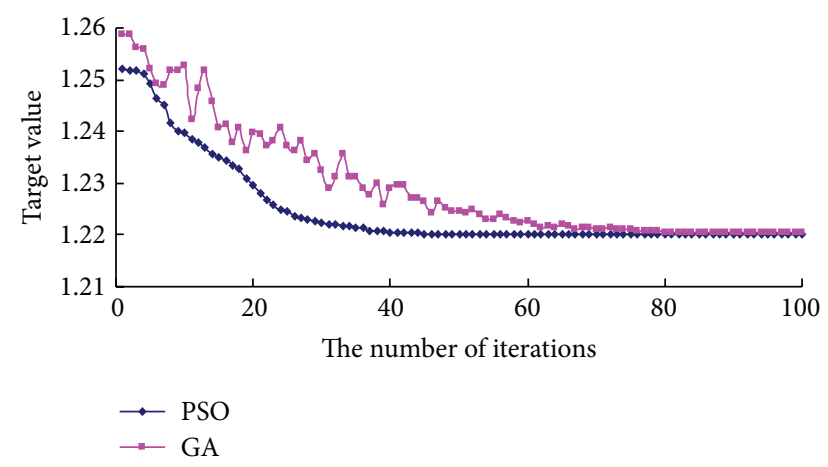

FIGURE 6: Iteration process.

the mechanical parameter, and the $Y$-axis is the important index. The $X$-axis factors $1-16$ correspond to the mechanical parameters in Table 3, respectively, where factors 1-8 are elastic modulus and factors 9-16 are strength parameters. Through Figure 4, it can be seen that the strength parameters have larger impact on the $K_{u}$ than the elastic modulus. The 12th parameter, RCC2 interface cohesion, has the greatest impact on the $K_{u}$, which indicates that the RCC2 interface is the most dangerous sliding plane of the dam failure.

5.3. Analysis of Evaluation Standard for RCCD Safety Coefficient. Through the above analysis, it is shown that the RCC2 interface is the most dangerous sliding plane of the RCCD. The quasielastic strength reserve coefficient of the RCC2 interface $K_{d}=1.76$ is obtained using SRF method in the design condition.

According to the previous analysis, the deterioration of strength parameters and seepage condition and the high water level will make the quasielastic strength reserve coefficient become smaller. So the strength parameters are chosen to be the minimums, the seepage condition is without drainage in dam foundation, the uplift pressure reduction factor $\alpha$ is 0.3 , and the upstream water level is 1.07 times higher than the design value. According to the test result, the ranges of the elastic modulus of the RCC bodies, conventional concrete, and dam foundation rock are shown in Table 4.
TABLE 6: Comparison between target values and estimated values from various algorithms for the training samples.

\begin{tabular}{lc}
\hline Criterion & MSE \\
\hline SWR & $3.35 e-6$ \\
LSSVM & $3.33 e-15$ \\
\hline
\end{tabular}

Adopting the LHS and SRF method, the quasielastic strength reserve coefficients corresponding to the combinations of the above deformation parameters are calculated, and the final results are shown in Table 5.

On this basis, the nonlinear relation between the deformation parameters and the quasielastic strength reverse coefficients is determined using the LSSVM. For the LSSVM, the ranges of parameters $\gamma$ and $\sigma$ are $\left[2^{-2}, 2^{60}\right]$ and $\left[2^{-2}, 2^{40}\right]$, respectively. LSSVM uses improved grid search algorithm to search the global best fitness value. The optimal solutions of parameters $\gamma$ and $\sigma$ obtained by rough search are $2^{54}$ and $2^{38}$, respectively. Then, a finer search is run in the region $\left[2^{52}, 2^{56}\right]$ and $\left[2^{36}, 2^{40}\right]$ for $\gamma$ and $\sigma$, respectively. The optimal solutions of the parameters $\gamma$ and $\sigma$ of the LSSVM using improved grid search algorithm are $2^{53}$ and $2^{39}$, respectively.

The stepwise regression (SWR) and LSSVM performances for training samples are shown in Table 6 and Figure 5. Table 6 shows the mean squares error (MSE) from PLSR and LSSVM for the training samples. Training result of the algorithms can be clearly seen in Figure 5. Table 6 and Figure 5 explain that the training performance of LSSVM is better than SWR.

PSO and Genetic Algorithm (GA) are applied to optimize the established LSSVM model. For the PSO, $c_{1}$ and $c_{2}$ all are chosen to be 2 , and the inertia weight $w$ decreases nonlinearly from 0.9 to 0.4 . For the GA, crossover probability and mutation probability are chosen to be 0.9 and 0.1 , respectively. Each algorithm uses 20 particles (or populations) to search the global best fitness value with each run executed for 200 iterations.

Figure 6 depicts the great success of optimization process using PSO compared with Genetic Algorithm (GA) for the quasielastic strength reserve coefficients $K_{m}$. It confirms the superiority of PSO in terms of convergence speed without the premature convergence problem. The minimums of the quasielastic strength reserve coefficients $K_{m}=1.22$ are obtained using two algorithms.

According to (24), the allowable value of the quasielastic strength reserve coefficients $\left[K_{e}\right]=1.31$ can be obtained. Due to $K_{d}=1.76>\left[K_{e}\right]=1.31$, it can be considered that the dam safety coefficient based on SRF method meets the requirement and the dam is safe in the design condition.

\section{Conclusions}

Evaluation standard for RCCD safety coefficient based on FEM is a problem requiring urgent solution. In this paper, the D-P criterion, SRF method, information entropy, and catastrophe theory are applied to RCCD failure criterion. On this basis, the LHS and auxiliary analysis of PLSR are used to 
determine the most dangerous sliding plane of RCCD failure. Finally, LHS, LSSVM, and PSO are adopted to establish evaluation standard for RCCD safety coefficient based on SRF method. The proposed method combines the advantages of several excellent algorithms and can be popularized to any RCCD. The analysis of evaluation standard for Longtan RCCD safety coefficient based on SRF method is carried out. The calculation shows that the ultimate failure is controlled by the strength of RCC 2 interface. In the design condition, the quasielastic strength reserve coefficient is 1.76 and the allowable value of the quasielastic strength reserve coefficient is 1.31. Due to $1.76>1.31$, the Longtan RCCD is safe in the design condition.

\section{Conflict of Interests}

The authors do not have any conflict of interests regarding the content of the paper.

\section{Acknowledgment}

The present work is supported by the Special Fund Projects supported by Basic Scientific Research Operating Expenses of Central-Level Public Academies and Institutes (no. CKSF2015022/GC, CKSF2014037/GC).

\section{References}

[1] F. Brian, "RCC-New developments and innovations," in Proceedings of the Brazilian International Roller Compacted Concrete (RCC) Symposium, pp. 1-18, Salvador, Brazil, 2008.

[2] Ministry of Water Resources of the People's Republic of China, Design Specification for Concrete Gravity Dams (SL-319-2005), China Water Power Press, Beijing, China, 2005.

[3] S. Q. Qin, "Primary discussion on formation mechanism of dissipative structure in instability process of rock mass," Chinese Journal of Rock Mechanics and Engineering, vol. 19, no. 3, pp. 265-269, 2000.

[4] M. D. McKay, R. J. Beckman, and W. J. Conover, "A comparison of three methods for selecting values of input variables in the analysis of output from a computer code," Technometrics, vol. 21, no. 2, pp. 239-245, 1979.

[5] A. M. J. Olsson and G. E. Sandberg, "Latin hypercube sampling for stochastic finite element analysis," Journal of Engineering Mechanics, vol. 128, no. 1, pp. 121-125, 2002.

[6] A. Olsson, G. Sandberg, and O. Dahlblom, "On Latin hypercube sampling for structural reliability analysis," Structural Safety, vol. 25, no. 1, pp. 47-68, 2003.

[7] F. Lindgren, P. Geladi, and S. Wold, "The kernel algorithm for PLS," Journal of Chemometrics, vol. 7, no. 1, pp. 45-59, 1993.

[8] M. Tenenhaus, V. E. Vinzi, Y. Chatelin, and C. Lauro, "PLS path modeling," Computational Statistics and Data Analysis, vol. 48, no. 1, pp. 159-205, 2005.

[9] J. A. K. Suykens and J. Vandewalle, "Least squares support vector machine classifiers," Neural Processing Letters, vol. 9, no. 3, pp. 293-300, 1999.

[10] J. Kennedy and R. Eberhart, "Particle swarm optimization," in Proceedings of the IEEE International Conference on Neural Networks, pp. 1942-1948, Perth, Australia, December 1995.
[11] A. Hasni, R. Taibi, B. Draoui, and T. Boulard, "Optimization of greenhouse climate model parameters using particle swarm optimization and genetic algorithms," Energy Procedia, vol. 6, pp. 371-380, 2011.

[12] D. C. Drucker and W. Prager, "Soil mechanics and plastic analysis or limit design," Quarterly of Applied Mathematics, vol. 10, pp. $157-165,1952$.

[13] Z. Wei, C. Xiaolin, Z. Chuangbing, and L. Xinghong, "Failure analysis of high-concrete gravity dam based on strength reserve factor method," Computers and Geotechnics, vol. 35, no. 4, pp. 627-636, 2008.

[14] O. C. Zienkiewicz and G. N. Pande, "Time-dependent multilaminate model of rocks-a numerical study of deformation and failure of rock masses," International Journal for Numerical and Analytical Methods in Geomechanics, vol. 1, no. 3, pp. 219-247, 1977.

[15] C. E. Shannon, "A mathematical theory of communication," The Bell System Technical Journal, vol. 27, pp. 379-423, 1948.

[16] C. Zhou and Y. Zhang, "Research on entropy catastrophic regularity and failure criterion in the deformation and failure process of rocks," Rock and Soil Mechanics, vol. 28, no. 12, pp. 2506-2510, 2007.

[17] C. Fu and S. Chen, "Study on instability criteria of surrounding rock of underground engineering cavern based on catastrophe theory," Rock and Soil Mechanics, vol. 29, no. 1, pp. 167-172, 2008.

[18] C. S. Gu, B. O. Li, G. L. Xu, and H. Yu, "Back analysis of mechanical parameters of roller compacted concrete dam," Science China Technological Sciences, vol. 53, no. 3, pp. 848-853, 2010.

[19] B. Li, C. Gu, Z. Li, and Z. Zhang, "Monitoring model for dam seepage based on partial least-squares regression and partial least square support vector machine," Shuili Xuebao, vol. 39, no. 12, pp. 1390-1400, 2008.

[20] B. Li, C. S. Gu, and J. K. Wu, "Gradual change law of elastic mechanical parameters of roller compacted concrete dam," Shuili Xuebao, vol. 44, no. 12, pp. 1488-1497, 2013.

[21] X. H. Ren, S. Y. Xia, and S. Q. Zhang, "Preliminarily study on failure mechanism of RCC gravity dams," Journal of Hohai University, vol. 25, no. 1, pp. 73-80, 1997.

[22] Y. M. Cheng, L. Li, S.-C. Chi, and W. B. Wei, "Particle swarm optimization algorithm for the location of the critical non-circular failure surface in two-dimensional slope stability analysis," Computers and Geotechnics, vol. 34, no. 2, pp. 92-103, 2007.

[23] X. Liu, C. Shao, H. Ma, and R. Liu, "Optimal earth pressure balance control for shield tunneling based on LS-SVM and PSO," Automation in Construction, vol. 20, no. 4, pp. 321-327, 2011.

[24] X. Wu, Q. Huang, and X. J. Zhu, “Thermal modeling of a solid oxide fuel cell and micro gas turbine hybrid power system based on modified LS-SVM," International Journal of Hydrogen Energy, vol. 36, no. 1, pp. 885-892, 2011.

[25] A. Alfi and H. Modares, "System identification and control using adaptive particle swarm optimization," Applied Mathematical Modelling, vol. 35, no. 3, pp. 1210-1221, 2011. 


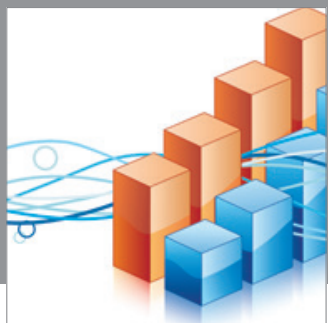

Advances in

Operations Research

mansans

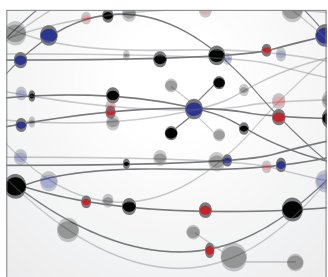

The Scientific World Journal
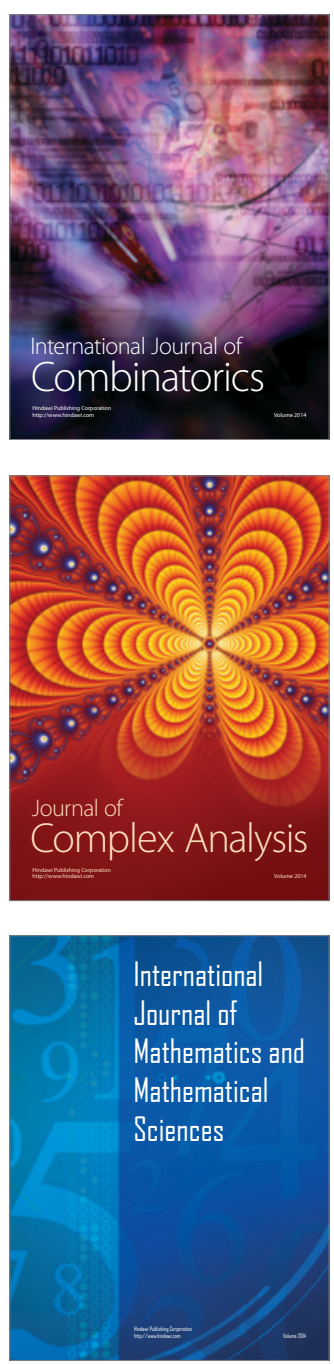
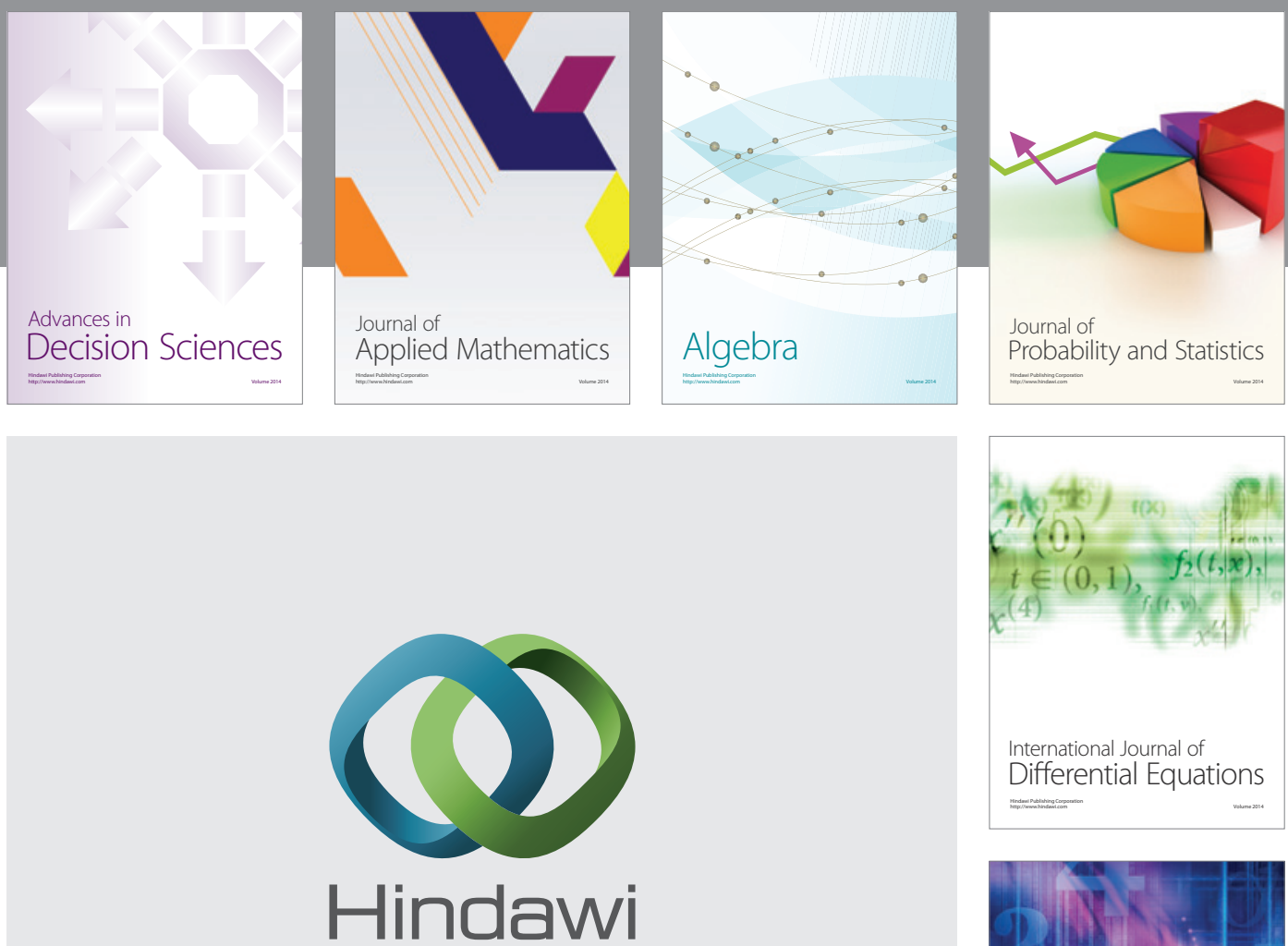

Submit your manuscripts at http://www.hindawi.com
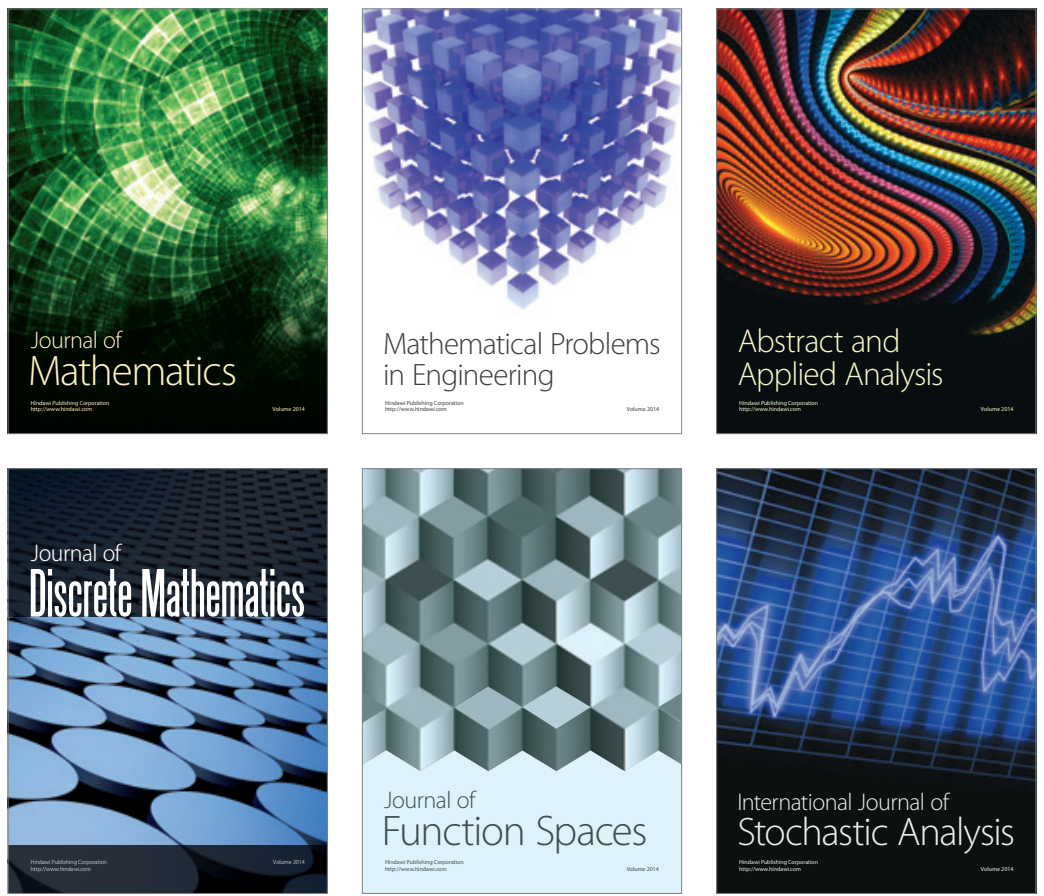

Journal of

Function Spaces

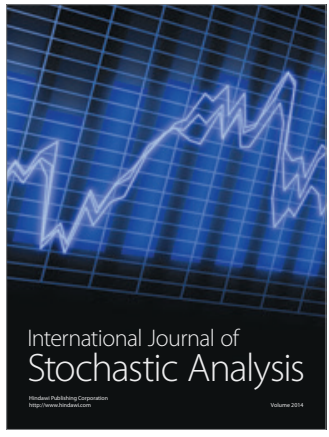

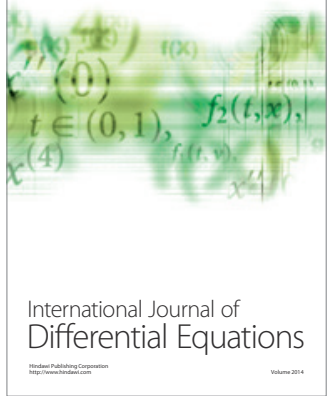
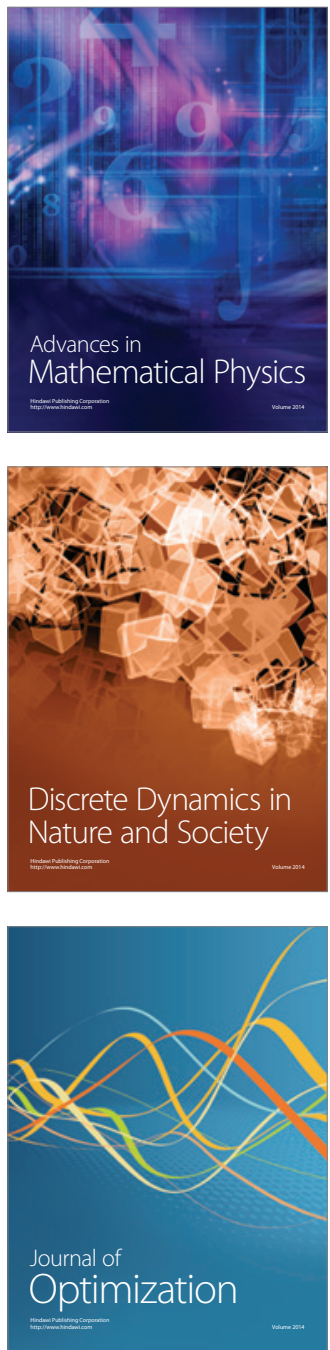